\title{
Sugar intake, serum insulin and platelet adhesiveness in men with and without peripheral vascular disease
}

\author{
JOHN YUDKIN \\ M.A., M.D., Ph.D. \\ Professor of Nutrition and Dietetics
}

STEPHEN SZANTo

Ph.D., M.R.C.P.I.

Research Clinician

Department of Nutrition, Queen Elizabeth College, University of London

\author{
V. V. KAKKAR \\ F.R.C.S.E., F.R.C.S. \\ Senior Surgical Registrar, \\ King's College Hospital Medical School
}

\begin{abstract}
Summary
Both the level of serum insulin and the adhesiveness of platelets are correlated with sucrose intake in men with peripheral vascular disease. These correlations do not exist in men who have no signs or symptoms of the disease, or of predisposing conditions such as hypertension.

The results support the suggestion of Szanto \& Yudkin (1969) that an habitual high intake of sugar raises the insulin level in some individuals but not all, and that these individuals are susceptible to the effect of sucrose in producing occlusive arterial disease.
\end{abstract}

\section{Introduction}

The reasons for supposing that a high intake of sucrose is the major dietary factor involved in the aetiology of ischaemic heart disease (IHD) have been summarized by Yudkin (1967a). A recent experiment in which nineteen young men were given diets high in sucrose for 2 weeks showed that only six responded by an increase in plasma insulin levels and platelet adhesiveness (Szanto \& Yudkin, 1969). From the results of this experiment, and from a consideration of the investigations of other workers, it was suggested that hyperinsulinism is an early manifestation-if not the primary manifestation-of IHD, that only some individuals in the population develop hyperinsulinism and increased platelet adhesiveness through a continuing high intake of sucrose, and that consequently only these will be susceptible to the effect of sucrose in producing IHD. We believe that these suggestions extend to persons with peripheral vascular disease, in whom we have shown a similar high intake of sucrose as in persons with IHD (Yudkin \& Roddy, 1964).

If this is correct, then individuals with occlusive arterial disease should have relatively high levels of insulin, and these would be expected to be related to their habitual intake of sugar. On the other hand, individuals with no obvious signs of occlusive arterial disease will include both those who are susceptible and those who are not. If we take a group of such apparently normal people, and exclude those who have or are likely to develop arterial disease, we should be left with a selected group composed mostly of those non-susceptible to sucrose. These, according to the hypothesis, will be those whose insulin levels are unaffected by their sugar intake.

Since the experimental high sucrose diet had produced an increase in platelet adhesiveness as well as increased insulin levels, we should expect the two groups of selected individuals to show the same relationship between platelet adhesiveness and sugar intake as that between insulin levels and sugar intake.

\section{Subjects and methods}

In the course of a continuing long-term study of diet in relation to occlusive arterial disease, we have been examining men and women attending the peripheral vascular clinic at King's College Hospital. At the same time, we have studied a group of apparently healthy men and women, of about the same age, who came for routine physical examination to the Medical Centre of the Institute of Directors. We should say at once that this latter group is not taken by us as a strict control group; for example, 
the individuals composing this group clearly do not have the same occupational distribution as those attending the hospital clinic. We should like to stress that this is a group of apparently healthy volunteers belonging to an economic class that is rarely used in clinical research. We do not, however, propose to compare directly the absolute values in one group with those in the other; what we shall compare is the correlation between sucrose consumption on the one hand, and insulin levels and platelet adhesiveness on the other hand, in the two groups.

Since there were few women in each group, we report here our findings only with the men. Amongst other investigations we have measured the level of serum immuno-reactive insulin after an overnight fast and during a glucose tolerance test after $50 \mathrm{~g}$ of oral glucose (Hales \& Randle, 1963), and we have measured fasting platelet adhesiveness (Wright, 1941). We have also assessed sugar intake by our routine questionnnaire (Yudkin, 1967b). From both groups of subjects, we have excluded all men who recorded that they had changed their sugar intake, and those who had manifest diabetes mellitus. From the directors' group, we also excluded men who showed hypertension or signs of IHD in their electrocardiographs. We were then left with twenty-seven men with peripheral vascular disease and twentyseven men who were apparently in normal health.

\section{Results}

The average age of the patients was 59 , with a range of 39-72; the average age of the directors was 51, with a range of 38-62. The relevant findings are given in Table 1. As we expected, the patients with vascular disease had higher insulin levels and higher platelet adhesiveness. We do not propose however to discuss the magnitude of these differences since, as we have pointed out, the two groups are not directly comparable; they differ for example not only in occupation but also to some extent in age.

We have calculated correlations for each group between sugar intake and insulin level and between sugar intake and platelet adhesiveness (Table 2). In the 'healthy' group of subjects, none of the correlations is statistically significant $(P>0.05)$.

In the group of patients with peripheral vascular disease, there is a significant correlation between sugar intake and insulin level, whether these were measured in the fasting state, or at 30 or $120 \mathrm{~min}$ after oral glucose. There is also a significant correlation between sugar intake and platelet adhesiveness; the value is negative because the method measures the proportion of platelets that do not adhere so that a low numerical value represents a high adhesiveness.

\section{Discussion}

We had not intended to compare directly the values of sucrose intake in the two groups, since our interest was only in the correlation between sucrose and insulin or platelet adhesiveness. By

TABLE 1. Findings in twenty-seven men with peripheral vascular disease and in twenty-seven apparently healthy men (mean values)

\begin{tabular}{lccccccc}
\hline & & Sugar & & \multicolumn{3}{c}{ Insulin $(\mu \mathrm{U} / \mathrm{ml})$} & \multicolumn{2}{c}{$\begin{array}{c}\text { Platelet } \\
\text { adhe }\end{array}$} & $\begin{array}{c}\text { A } \\
\text { intake }\end{array}$ & (years) & (g/day) & & 0 min & $30 \mathrm{~min}$ & $120 \mathrm{~min}$ & ness \\
\hline Patients with PVD & 59 & 123 & 25 & 81 & 56 & 62 \\
'Healthy' men & 51 & 119 & 19 & $48^{*}$ & $33^{*}$ & $6^{*}$ \\
\hline
\end{tabular}

*Significant difference $P<0.001$.

TABLE 2. Correlation between sugar intake, insulin level and platelet adhesiveness in twenty-seven men with PVD and twenty-seven 'healthy' men, before and during glucose tolerance test (tau values and $\boldsymbol{P}$ values for two-tailed test)

\begin{tabular}{|c|c|c|c|c|}
\hline \multirow{2}{*}{ Time of insulin assay } & \multicolumn{2}{|c|}{$\begin{array}{l}\text { Sugar intake and } \\
\text { insulin }\end{array}$} & \multicolumn{2}{|c|}{$\begin{array}{l}\text { Sugar intake and } \\
\text { platelet adhesiveness }\end{array}$} \\
\hline & Tau & $P$ & Tau & $P$ \\
\hline $\begin{array}{l}\text { A: Men with PVD } \\
\text { Fasting } \\
30 \text { min } \\
120 \mathrm{~min}\end{array}$ & $\begin{array}{l}0.30 \\
0.52 \\
0.34\end{array}$ & $\begin{array}{l}<0.05 \\
<0.001 \\
<0.02\end{array}$ & -0.28 & $<0.05$ \\
\hline $\begin{array}{l}\text { B: 'Healthy' men } \\
\text { Fasting } \\
30 \mathrm{~min} \\
120 \mathrm{~min}\end{array}$ & $\begin{array}{l}-0.03 \\
-0.19 \\
-0.06\end{array}$ & $\begin{array}{l}\text { NS } \\
\text { NS } \\
\text { NS }\end{array}$ & $-0 \cdot 18$ & NS \\
\hline
\end{tabular}




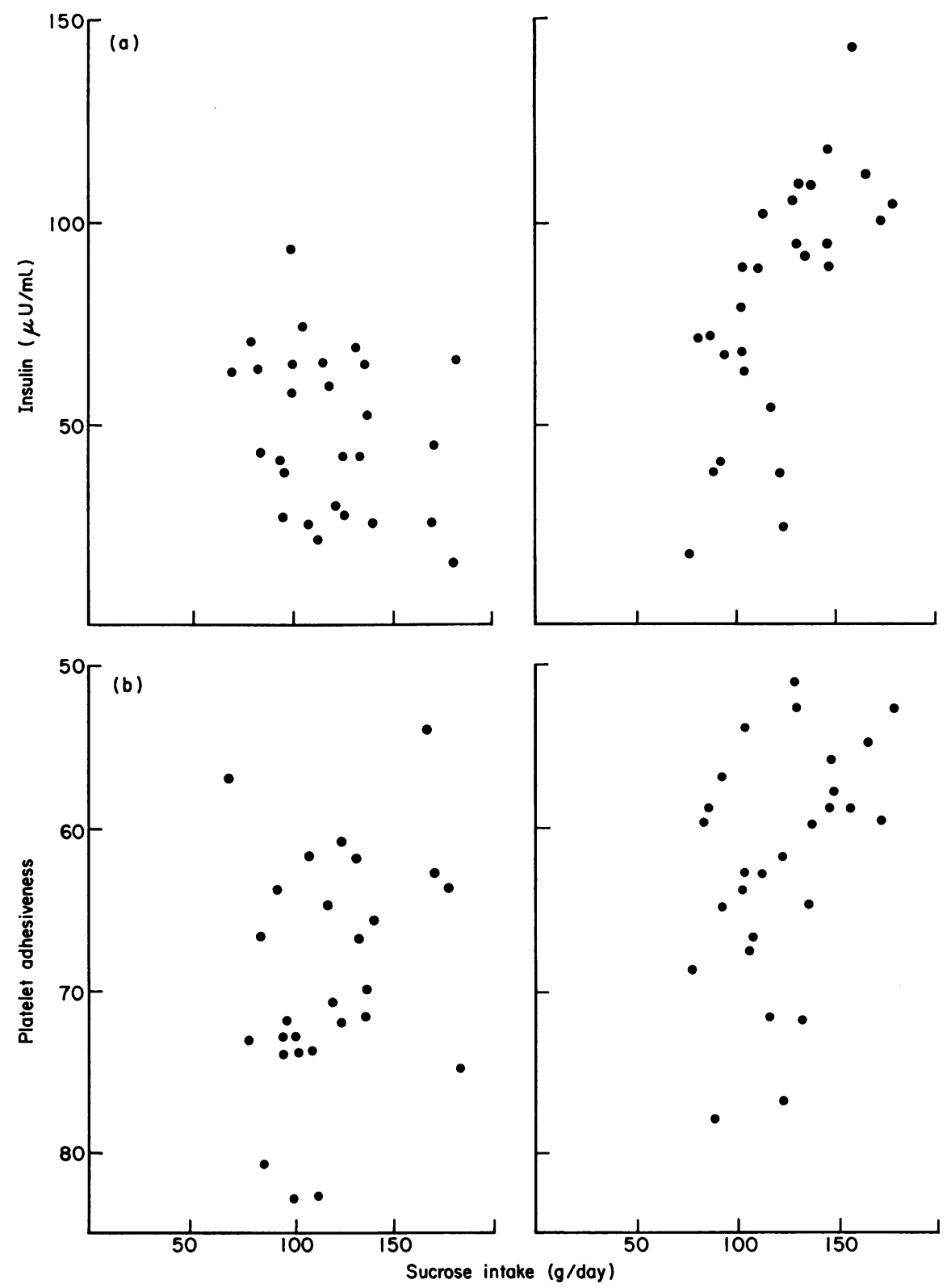

FIG. 1. Relationship between: (a) sugar consumption and immuno-reactive insulin ( $30 \mathrm{~min}$ after $50 \mathrm{~g}$ oral glucose), and (b) sugar consumption and platelet adhesiveness, in 'healthy' men (left-hand graphs) and in men with peripheral vascular disease (right-hand graphs).

chance however both the average sucrose consumption and its range were similar in the two groups. It is, therefore, clear at once that the correlation exists for the group of patients with vascular disease, but not for the healthy subjects (Fig. 1).

Since the two groups differed in occupation, we have to consider the possibility that there is an alternative explanation for our results. This explanation could be that intake of sucrose is correlated with levels of insulin and platelet adhesiveness in men who are mostly working class but not in men who are mostly professional. We think it safe to reject this alternative.

We can therefore say that our findings are in accord with the prediction that both insulin level and platelet adhesiveness would be related to habi- 
tual sugar intake in men with peripheral vascular disease, but not in men selected against the disease. These results are in conformity with the hypothesis of Szanto \& Yudkin (1969) that only some individuals in the population are susceptible to 'sucrose-induced hyperinsulinism', and that these are also susceptible to the development of occlusive arterial disease if they habitually consume high quantities of sucrose.

\section{Acknowledgments}

We are glad to record our thanks to the British Heart Foundation for a grant towards the cost of this study, and to Professor J. G. Murray and Dr Beric Wright for allowing us access to the subjects attending their clinics.

\section{References}

Hales, C.J. \& RaNDle, P.J. (1963) Immunoassay of insulin with insulin-antibody precipitate. Biochem. J. 88, 137.

Szanto, S. \& YudkIN, J. (1969) The effect of dietary sucrose on blood lipids, serum insulin, platelet adhesiveness and body weight in human volunteers. Postgrad. med. J. 45, 602.

WRIGHT, H.P. (1941) The adhesiveness of blood platelets in normal subjects with varying concentrations of anticoagulants. J. Path. Bact. 53, 255.

YUDKIN, J. (1967a) Why blame sugar? Chemy Ind. 1464.

YUDKIN, J. (1967b) Measurement of sugar consumption by questionary. Brit. med. J. 3, 154.

YUDKIN, J. \& RodDY, J. (1964) Levels of dietary sucrose in patients with occlusive atherosclerotic disease. Lancet, ii, 6. 